\title{
EXISTENCE AND STABILITY OF A CLASS OF NONLINEAR VOLTERRA INTEGRAL EQUATIONS
}

\author{
BY
}

STANLEY I. GROSSMAN

\begin{abstract}
In this paper we study the problem of existence and uniqueness to solutions of the nonlinear Volterra integral equation $x=f+a_{1} g_{1}(x)+\cdots+a_{n} g_{n}(x)$, where the $a_{1}$ are continuous linear operators mapping a Fréchet space $\mathscr{F}$ into itself and the $g_{i}$ are nonlinear operators in that space. Solutions are sought which lie in a Banach subspace of $\mathscr{F}$ having a stronger topology. The equations are studied first when the $g_{i}$ are of the form $g_{i}(x)=x+h_{i}(x)$ where $h_{i}(x)$ is "small", and then when the $g_{i}$ are slope restricted. This generalizes certain results in recent papers by Miller, Nohel, Wong, Sandberg, and Benex.
\end{abstract}

I. Introduction. The purpose of this paper is to study the behavior of solutions of the nonlinear Volterra integral equation

$$
x(t)=f(t)+\int_{0}^{t} a_{1}(t-s) g_{1}(x(s)) d s+\cdots+\int_{0}^{t} a_{n}(t-s) g_{n}(x(s)) d s .
$$

In §II these are studied in the abstract form

$$
x=f+a_{1} g_{1}(x)+\cdots+a_{n} g_{n}(x)
$$

where $x$ and $f$ are elements of a Fréchet space $\mathscr{F}$, the operators $a_{i}$ are linear continuous maps from $\mathscr{F} \rightarrow \mathscr{F}$ and the $g_{i}$ are nonlinear maps from $\mathscr{F} \rightarrow \mathscr{F}$. We assume that $g_{i}(x)=x+h_{i}(x)$ where the $h_{i}$ have certain "smallness" properties. Solutions are sought which lie in a Banach subspace $B$ of $\mathscr{F}$ with a stronger topology. By this we mean that convergence in $B$ implies convergence in $\mathscr{F}$.

In Theorem 2.1 we prove that the nonlinear problem (1.2) has a unique solution lying in $B$ if $f$ is in $B$ with small norm and there exists a bounded linear operator $\omega$ mapping $B \rightarrow B$, such that $a_{i}+\omega a_{i}$ is a bounded linear operator from $B \rightarrow B$ and $\left\|\sum a_{i}+\sum \omega a_{i}-\omega\right\|_{B}<1$.

Equations of this sort, with one kernel, have been studied by many authors;

Presented to the Society, January 22, 1970 under the title Global existance and uniqueness of solutions to Volterra integral equations; received by the editors July 3, 1969 and, in revised form, December 10, 1969.

AMS Subject Classifications. Primary 4513, 4530; Secondary 4780.

Key Words and Phrases. Volterra integral equation, nonlinear integral equation, applications of contraction map, integral equation resolvent, convolution equations, Fréchet space, completely monic function, Laplace-Stieltjes transform, multiple resolvent, slope restrictions, nonlinear network. 
in particular, Miller [4], Miller, Nohel, and Wong [5], Nohel [6], and Corduneanu [2]. It is shown in Corollary 2.4 and Theorem 2.10 that certain stability results established in [4] and [5] are special cases of the present results. We show further in Theorem 2.11 that if the linear equation

$$
y=f+a_{1} y+\cdots+a_{n} y
$$

has a solution in $B$ for every $f$ in $B$, then the same is true of the nonlinear equation (1.2) with $f$ restricted to a suitably small ball in $B$. Finally, in Theorems 2.2 and 2.7 we give applications of our theorems which were not obtainable by earlier results.

In §III, we impose slope restrictions on the nonlinearities to obtain similar results which are valid in a large class of spaces. Work in this area has been done by Beneš and Sandberg [1], Sandberg [8], and Zames [10] among others. The assumption here is that there exist $\alpha, \beta$ such that

$$
\alpha \leqq \frac{g(x, s)-g(y, s)}{x-y} \leqq \beta \text { for } x \geqq y \text { and } x, y \text { real. }
$$

This generalizes the work done in [1] and [7] in that the results are valid in a wider class of spaces (Beneš and Sandberg were interested in $L^{2}$ and $L^{\infty}$ ) and existence and uniqueness can be proven in a more general setting. That is, in Theorem 3.1 we show existence and uniqueness of solutions to the equation if a certain norm, defined in the hypotheses of the theorem, is $<1$ for any $f$ in $B$. In Corollary 3.2 we prove the same result for the case where the norm is $\geqq 1$ by restricting the class of functions $f$. The case of more than one kernel is also considered.

The Fréchet and Banach spaces that we will be particularly interested in are the following:

I. $\mathscr{F}=C[0, \infty)$ with the topology of uniform convergence on compact subsets of $[0, \infty)$. Then $B=B C[0, \infty)=\left\{x \in C[0, \infty): \sup _{t \geqq 0}|(x(t))|<\infty\right\}$ or

$$
B=B C_{0}[0, \infty)=\{x \in B C[0, \infty): x(t) \rightarrow 0 \text { as } t \rightarrow \infty\} .
$$

For either of these Banach subspaces we take as norm the sup norm.

II. $\mathscr{F}=L L^{p}[0, \infty)=\left\{x\right.$ measurable : $\int_{0}^{T}|x(t)|^{p}<\infty$ for all $\left.T>0\right\}$ with the topology of $L^{p}$ convergence on compact subsets of $[0, \infty)$. Then $B=L^{p}[0, \infty)$ with the usual $L^{p}$ norm, $1 \leqq P<\infty$.

Much of the research presented here appeared in the author's Ph.D. thesis (Brown University, 1969) under the direction of Professor R. K. Miller. This work was supported, in part, by the Air Force Office of Scientific Research under grant number AFOSR 67-0693A.

II. Equations with small nonlinearities. Let $\mathscr{F}$ be a Fréchet space, that is $\mathscr{F}$ is a complete metric space with metric $\rho$ such that 
(i) vector addition and scalar multiplication are $\rho$-continuous, and

(ii) $\rho(x, y)=\rho(x-y, 0)$.

Let $B$ be a Banach subspace of $\mathscr{F}$ with a stronger topology. We seek a solution to (1.2) lying in $B$. The following assumptions are needed.

(P1) For $i=1, \ldots, n, a_{i}$ is a continuous linear operator from $\mathscr{F} \rightarrow \mathscr{F}$.

(P2) $g_{i}(x)=x+h_{i}(x)$ where $h_{i}(x)$ maps $B \rightarrow B$ and has the property that for all $\varepsilon>0$, there exists $\delta>0$ such that if $\left\|x_{1}\right\|_{B},\left\|x_{2}\right\|_{B}<\delta$ then $\left\|g_{i}\left(x_{1}\right)-g_{i}\left(x_{2}\right)\right\|_{B} \leqq \varepsilon x_{1}-x_{2} \|_{B}$. We further assume that $h_{i}(0)=0$.

We note that if $h_{i}$ maps $B \rightarrow B$, is Fréchet differentiable and satisfies $\left\|h_{i}^{\prime}(x)\right\|_{B}$ $=O\left(\|x\|_{B}\right)$ as $\|x\|_{B} \rightarrow 0$, then $(\mathrm{P} 2)$ is satisfied.

(P3) $f \in B$.

(P4) There exists a function $\omega$ which belongs to $B L(B, B)$ (the set of bounded linear operators from $B \rightarrow B$ ) and satisfies:

(a) $a_{i}+\omega a_{i} \in B L(B, B)$ for all $i$.

(b) $\left\|\sum a_{i}+\sum \omega a_{i}-\omega\right\|_{B}<1$ (this is the operator norm).

(c) $(I-\omega)^{-1} \omega$ is a continuous operator mapping $\mathscr{F} \rightarrow \mathscr{F}$.

ReMarK. It is shown, in Theorem 2.10, that for the one kernel case, (P4) is equivalent to the condition that the integral equation resolvent is a bounded linear operator mapping $B \rightarrow B$.

The principal result of this section is the following:

THEOREM 2.1. If (P1)-(P4) are satisfied, there exist $\varepsilon_{1}$ and $\varepsilon_{2}>0$ such that if $\|f\|_{B}<\varepsilon_{1}$, there is a unique solution $x$ to (1.2) which lies in $B$ and $\|x\|_{B}<\varepsilon_{2}$.

Proof. Define a vector $v$ by $v=-(I+\omega)^{-1} \omega$. Then $-\omega=v+\omega v$ or $(I+v)(I+\omega)$ $=I$. Thus $(I+v)^{-1}=(I+\omega)$. Here $I$ is the identity operator in the Fréchet space. We now add $v x$ to both sides of (1.2) to obtain

$$
x+v x=f+v x+\sum_{i=1}^{n} a_{i} x+\sum_{i=1}^{n} a_{i} h_{i}(x)
$$

Multiplying both sides by $(I+v)^{-1}$, we obtain

$$
x=f+\omega f+\omega v x+\sum_{i=1}^{n} \omega a_{i} x+\sum_{i=1}^{n} a_{i} h_{i}(x)+\sum_{i=1}^{n} \omega a_{i} h_{i}(x)+v x+\sum_{i=1}^{n} a_{i} x .
$$

We note that $(I+\omega) v x=(v+\omega v) x=-\omega x$. If we let $B_{i}=a_{i}+\omega a_{i}$, the equation becomes

$$
x=\hat{f}+\left[\sum B_{i}-\omega\right] x+\sum B_{i} h_{i}(x) \equiv T x .
$$

Here $\hat{f}$ denotes $f+\omega f$ which lies in $B$.

To complete the proof we show that $T$ maps $B \rightarrow B$ and that $T$ is a contraction. We note that $f \in B, x \in B$ implies that $h_{i}(x) \in B$ by (P2). Also $B_{i}$ and $\omega \in B L(B, B)$ by (P4) so $T$ maps $B \rightarrow B$. Now, we let $R=\sum_{i=1}^{n} B_{i}-\omega, k=\|R\|_{B}<1, c_{i}=\left\|B_{i}\right\|_{B}$ 
$=\left\|a_{i}+\omega a_{i}\right\|_{B}$ and choose $\delta=(1-k) /\left(2 \sum_{1}^{n} c_{i}\right), \varepsilon_{2}=\varepsilon(\delta)$ and $\varepsilon_{1}=((1-k) / 2) \varepsilon_{2} /\|I+\omega\|_{B}$. Finally we define $S\left(0, \varepsilon_{2}\right)=\left\{x \in B:\|x\|_{B} \leqq \varepsilon_{2}\right\}$. Now, if $x_{1}$ and $x_{2} \in S$,

$$
\begin{aligned}
\left\|T x_{1}-T x_{2}\right\| & \leqq\left\|R\left(x_{1}-x_{2}\right)\right\|+\left\|\sum B_{i}\left(h_{i}\left(x_{1}\right)-h_{i}\left(x_{2}\right)\right)\right\| \\
& \leqq\|R\|\left\|x_{1}-x_{2}\right\|+\sum\left\|B_{i}\right\|\left\|h_{i}\left(x_{1}\right)-h_{i}\left(x_{2}\right)\right\| \\
& \leqq k\left\|x_{1}-x_{2}\right\|+\sum c_{i} \frac{(1-k)}{2 \sum c_{i}}\left\|x_{1}-x_{2}\right\| \\
& =\left(k+\frac{1-k}{2}\right)\left\|x_{1}-x_{2}\right\|<\left\|x_{1}-x_{2}\right\|
\end{aligned}
$$

so that $T$ is contracting. To show that $T$ maps $S \rightarrow S$, let $x \in S$ so that

$$
\begin{aligned}
\|T x\| & \leqq\|\hat{f}\|+k\|x\|+\sum c_{i}\left\|h_{i}(x)\right\| \\
& =\|\hat{f}\|+k\|x\|+\sum c_{i}\left\|h_{i}(x)-h_{i}(0)\right\| \\
& \leqq\|\hat{f}\|+k\|x\|+\frac{1-k}{2}\|x\| \leqq\left(\frac{1-k}{2}+k+\frac{1-k}{2}\right) \varepsilon_{2}=\varepsilon_{2} .
\end{aligned}
$$

REMARK. For equation (1.1), condition (P4)(c) is automatically satisfied if $B$ is any of the spaces $B C[0, \infty)$ or $L^{p}[0, \infty), 1 \leqq p \leqq \infty$. We then require that there exist an $L L^{1}$ solution to the equation

$$
v(t)=-\omega(t)-\int_{0}^{t} \omega(t-s) v(s) d s \quad \text { where } \quad \omega(t) \text { is in } L^{1}[0, \infty) .
$$

Such a solution is easily shown to exist by standard integral equation arguments. Also, for the above-mentioned spaces conditions (P4)(a) and (P4)(b) can be restated as follows:

(P4') There exist $\omega \in L^{1}[0, \infty)$ such that:

(a) $a_{i}+a_{i} * \omega \in L^{1}[0, \infty), i=1, \ldots, n$ (* denotes convolution),

(b) $\left\|\sum a_{i}+\sum a_{i} * \omega-\omega\right\|_{1}<1$.

Condition (P1) can be replaced by $\left(\mathrm{P}^{\prime}\right) a_{i} \in L L^{1}[0, \infty), i=1, \ldots, n$.

It should also be pointed out that it is not necessary to consider these equations in convolution form. If, for example, $x(t)=f(t)+\int_{0}^{t} a(t, s) g(x(s)) d s$, then it is shown in [5] that the condition that $B=a+a * \omega \in B L(B, B)$ could be replaced by the condition that $B x=\int_{0}^{t} b(t, s) x(s) d s$ represents a bounded linear operator from $B \rightarrow B$. If $B=B C[0, \infty)$, for example, then $b(t) \in L^{1}[0, \infty)$ is replaced by the following condition:

There exists a constant $k>0$ such that

$$
\int_{0}^{t}|b(t, s)| d s \leqq A \text { for all } t \geqq 0 .
$$

We should also point out that the condition $f \in B$ can be relaxed. The only thing needed in the proof of Theorem 2.1 is that $(I+\omega) f=\hat{f} \in B$. We then require that $\hat{f}$ is "small" (that is, $\|\hat{f}\|<\varepsilon_{1}$ ) to get the same result. 
Finally, we note that if the $h_{i}$ 's have global Lipschitz constants sufficiently small so as to make $T x$ a contraction, it is then not necessary to restrict $f$ to a small ball in $B$. Any $f$ will give a unique solution to (1.2).

EXAMPLE 2.1. Let

$$
x(t)=f(t)+\int_{0}^{t} a(t-s) g(x(s)) d s
$$

where $a(t)=-1+e^{-t} \notin L^{1}[0, \infty)$. We assume that $f$ and $g$ satisfy the other conditions of Theorem 2.1. Let $F=C[0, \infty)$ and $B=B C[0, \infty)$. Clearly $a(t)$ satisfies (P1), but does not map $B \rightarrow B$ since $a(t) \notin L^{1}[0, \infty)$. Let $\omega(t)=-e^{-t} \in L^{1}[0, \infty)$. Then

$$
\begin{aligned}
a * \omega(t) & =-\int_{0}^{t} e^{-(t-s)}\left(-1+e^{-s}\right) d s=-e^{-t} \int_{0}^{t} e^{s}\left(-1+e^{-s}\right) d s \\
& =1-e^{-t}-t e^{-t} \\
a(t)+a * \omega(t) & =-t e^{-t} \in L^{1}[0, \infty) \\
a+a * \omega-\omega & =e^{-t}-t e^{-t}=e^{-t}(1-t)
\end{aligned}
$$

and finally

$$
\|a+a * \omega-\omega\|=\int_{0}^{1} e^{-t}-t e^{-t}+\int_{1}^{\infty} t e^{-t}-e^{-t}=\frac{2}{e}<1 .
$$

A general class of examples can be found which satisfy the conditions of Theorem 2.1. Let $z(t)$ be any $L^{1}$ function having the property that $\left|z\left(t_{1}+t_{2}\right)\right| \leqq\left|z\left(t_{1}\right)\right|\left|z\left(t_{2}\right)\right|$. The prototype of course is $e^{-t}$. We assume, for simplicity, that $\|z\|_{1} \leqq 1$. If not, a norming factor is easily introduced in what follows. We define the space $L_{z}=$ $\left\{x\right.$ measurable: $\left.\int_{0}^{\infty}|x(t)||z(t)| d t<\infty\right\}$. For $x \in L_{z}$, we define

$$
\|x\|_{z}=\int_{0}^{\infty}|x(t)||z(t)| d t
$$

Clearly $\|\cdot\|_{z}$ is a norm. Also $L_{z}$ is a Banach space since $L_{z}$ is the set of functions which are $L_{1}$ with respect to the measure $d \mu=|z(t)| d t$. As before, we define

$$
L L_{z}=\left\{x \text { measurable: } \int_{0}^{T}|x(t)||z(t)| d t<\infty \text { for all } T>0\right\}
$$

THEOREM 2.2. Suppose (P1)-(P3) are satisfied for $F=L L_{z}$ and $B=L_{z}$. Suppose $a_{i}(t) \in L L_{1}[0, \infty)$ and there exits $\omega(t)$ such that $\sum a_{i}+\sum a_{i} * \omega-\omega \in L_{\infty}[0, \infty)$ and $\left\|\sum a_{i}+\sum a_{i} * \omega-\omega\right\|_{\infty}<1$. Then the conclusions of Theorem 2.1 hold.

Proof. We show that $T=\sum a_{i}+\sum a_{\mathfrak{i}} * \omega-\omega$ is a bounded linear operator from $L_{z} \rightarrow L_{z}$ and $\|T\|_{z}<1$. For $x \in L_{z}$, 


$$
\begin{aligned}
\|T x\|_{z} & =\int_{0}^{\infty}\left|\int_{0}^{t} T(t-s) x(s) d s\right||z(t)| d t \\
& \leqq \int_{0}^{\infty} \int_{0}^{t}|T(t-s)||x(s)||z(t)| d s d t=\int_{0}^{\infty} \int_{s}^{\infty}|T(t-s)||x(s)||z(t)| d t d s \\
& =\int_{0}^{\infty}|x(s)| \int_{0}^{\infty}|T(u)||z(u+s)| d u d s \\
& \leqq \int_{0}^{\infty}|T(u)||z(u)| d u \int_{0}^{\infty}|x(s)||z(s)| d s \\
& \leqq\|T\|_{\infty}\|z\|_{1}\|x\|_{z} \leqq\|T\|_{\infty}\|x\|_{z}
\end{aligned}
$$

and so

$$
\sup _{\|x\|_{z} \leqq 1}\|T x\|_{z} /\|x\|_{z} \leqq\|T\|_{\infty}<1 .
$$

EXAMPLE 2.2 Let $a_{1}(t)=(t+2)^{-1 / 2}, a_{2}(t)=\cdots=a_{n}(t)=0, z(t)=e^{-t}$. Choose

$$
\begin{aligned}
\omega(t)=-\chi_{[0,1]}(t) & =-1 & & 0 \leqq t \leqq 1, \\
& =0 & & \text { otherwise. }
\end{aligned}
$$

Then

$$
a * \omega(t)=-\int_{0}^{t}(t-s+2)^{-1 / 2}=-2\left((t+2)^{1 / 2}-(t+1)^{1 / 2}\right)=-(t+\delta)^{-1 / 2}
$$

where $1<\delta<2$ by the mean value theorem. Then

$$
a+a * \omega=\frac{1}{(t+2)^{1 / 2}}-\frac{1}{(t+\delta)^{1 / 2}}=-\frac{(2-\delta)}{2\left(t+\delta^{\prime}\right)^{3 / 2}}, \quad 1<\delta<\delta^{\prime}<2,
$$

and $a+a * \omega-\omega=\chi_{[0,1]}(t)-(2-\delta) /\left(2\left(t+\delta^{\prime}\right)^{3 / 2}\right) \equiv T$. Thus

$$
\begin{aligned}
\|T\|_{\infty} & =\max \left\{\sup _{0 \leqq t \leqq 1} 1-\frac{2-\delta}{2\left(t+\delta^{\prime}\right)^{3 / 2}}, \sup _{1<t} \frac{2-\delta}{2\left(t+\delta^{\prime}\right)^{3 / 2}}\right\} \\
& =1-\frac{2-\delta}{2\left(1+\delta^{\prime}\right)^{3 / 2}}<1
\end{aligned}
$$

since $1<\delta<\delta^{\prime}<2$ and Theorem 2.2 applies in the space $L L_{z}$. In particular, with $z(t)=e^{-t}$, we have shown that unique solution to (1.1) cannot grow faster than an exponential. The next example illustrates Theorem 2.1 with more than one kernel.

ExAmple 2.3. Let $n=2$ and let $a_{1}(t)=-1+e^{-t}, a_{2}(t)=-1+e^{-2 t}$. Again, let $\omega(t)=-e^{-t}$. Then, as in Example 2.1, $a_{1}+a_{1} * \omega(t)=-t e^{-t}$ and $a_{2}+a_{2} * \omega(t)$ $=2 e^{-2 t}-2 e^{-t}$. Then $a_{1}(t)+a_{1} * \omega(t)+a_{2}(t)+a_{2} * \omega(t)-\omega(t)=2 e^{-2 t}-e^{-t}-t e^{-t}$ $\in L^{1}[0, \infty)$. Call this function $S(t)$. We must check that $\|S\|_{1}<1$. This is a difficult calculation since $S(t)$ changes sign. In order to determine the norm one must solve the transcendental equation $2 e^{-2 t}=e^{-t}+t e^{-t}$. For this reason, we leave the example for the time being and return to it later with a simpler method of solution. 
An easy stability result can be derived from Theorem 2.1. We can show that, under suitable conditions, the system (1.1) is asymptotically stable in the sense that if the forcing function $f(t)$ is bounded, continuous, and tends to zero with increasing time, then the same is true of the solution $x(t)$. This is shown in [5] for the one kernel case.

If we let $B_{0}=\{x \in B C[0, \infty): x(t) \rightarrow 0$ as $t \rightarrow \infty\}$ it is clear that $B_{0}$ is a Banach space with the sup norm.

COROllaRY 2.3. Under assumptions (P1)-(P4) with $B=B_{0}$ then there is a unique solution to (1.1) lying in $B_{0}$ for $f \in B_{0}$ and $\|f\|_{0}$ small enough.

Proof. Obvious.

In recent papers Miller [4], and Miller, Nohel, and Wong [5] have explored a linearization technique for nonlinear Volterra integral equations in several different spaces with one kernel. We now show that certain of these results are a corollary to Theorem 2.1 and that they can be generalized to more than one kernel. Consider the equation

$$
x(t)=f(t)+\int_{0}^{t} a(t-s) k(x(s)) d s .
$$

For this equation we define the resolvent of $a(t)$ (denoted res $a$ ) by the resolvent equation

$$
r(t)=-a(t)+\int_{0}^{t} a(t-s) r(s) d s .
$$

Then $(I-r) *(I-a)=I-a-r+a r=I$ or $(I-r)=(I-a)^{-1}$.

COROLlaRY 2.4. Suppose (P1)-(P3) are satisfied for equation (2.2) and suppose that res $a \in B L(B, B)$. Then the conclusions of Theorem 2.1 hold.

Proof. Let $\omega(t)=-r(t)$. Then $a+a * \omega=a-a * r=-r(t) \in B L(B, B)$ and $\|a+a * \omega-\omega\|_{B}=0<1$. Q.E.D.

The following result is due to Paley and Wiener [7] and is useful when $a+a * \omega$ $\in B L(B, B)$ is satisfied when $a+a * \omega \in L^{1}[0, \infty)$.

Lemma 2.5. If $f \in L^{1}[0, \infty)$ and the Laplace transform $\hat{f}(s) \neq 1$ for $\operatorname{Re} s \geqq 0$, then res $f \in L^{1}[0, \infty)$. Here $\hat{f}(s)=\int_{0}^{\infty} e^{-s t} f(t) d t$.

We can use this result to sharpen Theorem 2.1. It may be possible, for example, to find an $\omega$ which satisfies $a+a * \omega \in B L(B, B)$ but the norm of $a+a * \omega-\omega$ is difficult to calculate. This was the case for Example 2.3. We thus have the following result.

Corollary 2.6. Suppose (P1)-(P3), and (P4') (a) are satisfied. Suppose further that $\hat{O}(s) \neq 1$ for $\operatorname{Re} s \geqq 0$ where $U=\sum a_{i} * \omega+\sum a_{i}-\omega$. Then the conclusions of Theorem 2.1 hold. 
Proof. Equation (1.1) can be written as (2.1) where $U=\sum B_{i}-\omega$. That is, $x=(f+\omega f)+U * x+$ "small terms". The hypotheses imply that res $U \in L^{1}[0, \infty)$ so we can apply Corollary 2.4. Q.E.D.

EXAMPLE 2.4 (CONTINUED). $U=a_{1}+a_{1} * \omega+a_{2} * \omega-\omega=2 e^{-2 t}-e^{-t}-t e^{-t}$. Thus

$$
\hat{O}(s)=\frac{2}{s+2}-\frac{1}{s+1}-\frac{1}{(s+1)^{2}}
$$

We must have $\hat{U}(s)=1$ only if $\operatorname{Re} s<0$. Thus, if $\hat{U}(s)=1$,

$$
\frac{2}{s+2}-\frac{1}{s+1}-\frac{1}{(s+1)^{2}}=1
$$

and simplifying we have $s^{3}+3 s^{2}+5 s+4=P(s)$. If $s_{1}, s_{2}$, and $s_{3}$ are the roots of $P(s)$, then by Vieta's formula, $\sum_{1}^{3} s_{i}=-a_{1} / a_{0}=-3$. Now, $P(-1)=1$ and $P(-2)=-2$ so there is a root between -1 and -2 . The sum of the other two roots therefore must be negative and since there obviously are no real positive roots, we must have all roots with negative real parts. Q.E.D.

The following corollary gives a general class of kernels which satisfy (P4), and will include Example 2.1.

THEOREM 2.7. Let $a(t)=-b(t)+c(t)$ where $c(t) \in L^{1}[0, \infty),\|c\|_{1} \leqq 1$ and $b(t)$ satisfies:

(i) $b(t) \in L^{1}[0,1], b(t) \not \equiv 0$

(ii) $b(t)$ is completely monic on the interval $0 \leqq t<\infty$, i.e. $b(t) \in C^{\infty}[0, \infty)$ and $(-1)^{j} b^{(j)}(t) \geqq 0$ for $j=0,1,2,3, \ldots$

Then there exists $\omega \in L^{1}[0, \infty)$ which satisfies (P4).

Proof. We wish to find $\omega \in L^{1}[0, \infty)$ such that $a+a * \omega \in L^{1}[0, \infty)$ and $\|a+a * \omega-\omega\|_{1}<1$. It suffices to find a solution of

$$
x=a+a * x
$$

which lies in $L^{1}[0, \infty)$. Rewriting (2.3), we have

$$
x+b * x=a+c * x .
$$

It follows from Theorem 2 and Corollary 2 of [3] that if $r(t)=$ res $(-b(t))$, then $0 \leqq r(t) \leqq b(t), \int_{0}^{\infty} r(t) d t \leqq 1$ and $r(t) \not \equiv 0$ on any interval of the form $(0, T), T>0$. Thus (2.4) becomes

$$
x=(I-r) * a+[(I-r) * c] * x .
$$

Now, $(I-r) * a=-b+b * r+c-c * r=r+c-c * r \in L^{1}[0, \infty)$. Let

$$
s=\operatorname{res}[(I-r) * c] \text { and } f=(I-r) * a \in L^{1}[0, \infty) \text {. }
$$

We then have

$$
x=(I-s) * f
$$


To show that $x \in L^{1}[0, \infty)$, it suffices to show that $s(t) \in L^{1}[0, \infty)$ or, equivalently, that $[(I-r) * c(s)]^{\wedge} \neq 1$ for $\operatorname{Re} s \geqq 0$. Now

$$
\left\|[(I-r) * c]^{\wedge}\right\| \leqq|1-\hat{r}(s)||\hat{c}(s)| \leqq|1-\hat{r}(s)|\|c\|_{1} \leqq|1-\hat{r}(s)|
$$

since $\|c\|_{1} \leqq 1$. We now show that for $\operatorname{Re} s \geqq 0,|1-\hat{r}(s)|<1$. For this, we need the following lemmas whose proofs can be found in Widder [9].

LEMMA 2.8. A function $f$ is completely monic on the interval $0 \leqq t<\infty$ if and only if $f$ is the Laplace-Stieltjes transform of a nondecreasing function $\alpha$; that is

$$
f(t)=\int_{0}^{\infty} e^{-t u} d \alpha(u)
$$

Note that the integrand in (2.7) is nonnegative so that the integral (2.8) exists for any real or complex $t$ with $\operatorname{Re} t>0$.

LEMMA 2.9. Let $f(t)=\int_{0}^{\infty} e^{-t u} d \alpha(u)$. Then the Laplace transform

$$
\hat{f}(s)=\int_{0}^{\infty} d \alpha(t) /(s+t) .
$$

$(\hat{f}(s)=s\{\alpha\}$ is the Stieltjes transform of $\alpha$.

Now, let $s=0$; then $\hat{r}(0)=\int_{0}^{\infty} r(t) d t \leqq 1$ so the transform is deflned and continuous at $s=0$. Since $r(t) \rightarrow 0$ as $t \rightarrow \infty, \hat{r}(s)$ can be extended analytically to the half plane $\operatorname{Re} s>0$. Returning to the definition of $r$, we have $r(t)=b(t)-$ $\int_{0}^{t} b(t-s) r(s) d s$ or $\hat{r}=\hat{b} /(1+\hat{b})$. Thus, $|1-\hat{r}(s)|=|1-\hat{b}(s) /(1+\hat{b}(s))|=|1 /(1+\hat{b}(s))|$. We must therefore show that $1<|1+\hat{b}(s)|$. It suffices to show that $\operatorname{Re} \hat{b}(s)>0$ for $\operatorname{Re} s \geqq 0$. But, by Lemmas 2.8 and $2.9, \hat{b}(s)=\int_{0}^{\infty} d \alpha(t) /(t+s)$ where $d \alpha(t)$ is nonnegative. Hence, for $\operatorname{Re} s \geqq 0, \operatorname{Re} \hat{b}(s)=\operatorname{Re} \int_{0}^{\infty} d \alpha(t) /(t+s)$. Let $s=\delta+\gamma i$. Then

$$
\begin{aligned}
\operatorname{Re} \int_{0}^{\infty} \frac{d \alpha(t)}{t+\delta+\gamma i} & =\operatorname{Re} \int_{0}^{\infty} \frac{(t+\delta-\gamma i) d \alpha(t)}{(t+\delta)^{2}+\gamma^{2}} \\
& =\int_{0}^{\infty} \frac{(t+\delta) d \alpha(t)}{(t+\delta)^{2}+\gamma^{2}}>0 \text { for } \delta=\operatorname{Re} s>0 . \quad \text { Q.E.D. }
\end{aligned}
$$

Functions which satisfy the condition on $b(t)$ as in Theorem 2.7 are numerous. For example, $b(t)=-c$ where $c$ is a positive constant, or $b(t)=-1 /(t+k)^{\delta}$ where $k, \delta>0$.

We now show that, in the one kernel case, Theorem 2.1 and Corollary 2.4 are essentially equivalent.

TheOREM 2.10. Let $x=f+a * k(x)$. Then res $a \in B L(B, B)$ if and only if there exists $\omega \in B L(B, B)$ such that $a+a * \omega \in B L(B, B)$ and $\|a+a * \omega-\omega\|_{B}<1$.

Proof. Suppose res $a \in B L(B, B)$. Then $\omega=-r$ works. Now suppose there exists $\omega$ such that $a+a * \omega \in B L(B, B)$ and $\|a+a * \omega-\omega\|_{B}<1$. Since $B L(B, B)$ is a Banach algebra (with multiplication defined by composition) and since 
$\|a+a * \omega-\omega\|_{B}<1$, it follows that $[I-(a+a * \omega-\omega)]^{-1} \in B L(B, B)$. Now, $r=$ $-a+a * r$ or $-a *(I-r)=r$ or $a=-(I-r)^{-1} * r$. Let $s=a+a * \omega$. Then

$$
a+a * \omega=-(I-r)^{-1} * r-(I-r)^{-1} * r * \omega=s \in B L(B, B) .
$$

Thus, multiplying by $(I-r)$, we have

$$
-r-r * \omega=(I-r) * s=s-r * s
$$

or

$$
r * s-r-r * \omega=s
$$

which implies that

$$
s=-r *(-s+I+\omega) .
$$

But $-s+I-\omega=I-(a+a * \omega-\omega)$ which is invertible. Thus

$$
-r=[I-(a+a * \omega-\omega)]^{-1} * s \in B L(B, B) .
$$

Q.E.D.

In the one kernel case, the resolvent of the kernel does not in any way depend on the nonlinear terms. When the resolvent belongs to $B L(B, B)$, we always will have a unique solution to the nonlinear equation if the forcing function has a sufficiently small norm. We now show that if there is a unique solution to the linear equation in $B$, then there will exist a unique solution in $B$ to the nonlinear equation if we restrict the forcing function $f(t)$ to a suitably small ball about the origin in $B$. The linear equation is given by

$$
y(t)=f(t)+\int_{0}^{t} a(t-s) y(s) d s .
$$

THEOREM 2.11. Assume (P1)-(P3) and suppose there exists a unique solution to (2.8) lying in $B$ for every $f$ in $B$. Then the conclusions of Theorem 2.1 hold.

Proof. We show that the hypotheses imply that res $a \in B L(B, B)$. For every $f \in B$, there exists $y \in B$ such that $y=f+a * y$. Thus $y-a * y=f$ or $y=(I-a)^{-1} * f$. This defines $(I-a)^{-1}$. Since $a$ is continuous, one to one, and onto in $F,(I-a)^{-1}$ is defined and continuous as a mapping from $F \rightarrow F$. Thus $(I-a)^{-1}$ is closed as a mapping from $B \rightarrow B$. Since the domain of $(I-a)^{-1}$ is all of $B,(I-a)^{-1}$ is continuous as a mapping from $B \rightarrow B$ by the closed graph theorem. Thus $(I-a)^{-1}$ $\in B L(B, B)$. Now, $a=I-(I-a)$ which implies that $(I-a)^{-1} * a=(I-a)^{-1}-I$ and so $(I-a)^{-1} * a \in B L(B, B)$. But $(I-a)^{-1} * a=-$ res $a$ from which the conclusion follows. Q.E.D.

We now can easily generalize the results of Corollary 2.4 to more than one kernel. We define the multiple resolvent of $a_{1}, \ldots, a_{n}$ follows:

$$
r(t)=-\sum a_{i}(t)+\int_{0}^{t} \sum a_{i}(t-s) r(s) d s .
$$


Corollary 2.12. Assume (P1)-(P3) hold and that $r(t)$ as defined above is in $B L(B, B)$. Suppose further that $a_{i}+a_{i} * r \in B L(B, B)$ for all $i$. Then the conclusions of Theorem 2.1 hold.

Proof. Let $\omega(t)=-r(t)$. Q.E.D.

III. Equations with slope restriction. In this section we make other assumptions about the nonlinearities. This work was motivated by that done by Beneš and Sandberg [1] and Sandberg [8] who impose slope restrictions on the nonlinear terms.

We begin with consideration of the one kernel case. Later, this is extended to the more general situation.

Consider the equation

$$
x(t)=f(t)+\int_{0}^{t} a(t-s) g(x(s), s) d s .
$$

We need the following assumptions:

(P7) There exist constants $\alpha$ and $\beta(\beta>0)$ with $\alpha<\beta$ such that

$$
\alpha\left(u_{1}-u_{2}\right) \leqq g\left(u_{1}, t\right)-g\left(u_{2}, t\right) \leqq \beta\left(u_{1}-u_{2}\right)
$$

for $u_{1} \geqq u_{2}$ and $u_{1}, u_{2}$ real.

(P8) There exists a function $\omega(t)$ in $L^{1}[0, \infty)$ such that $a+a * \omega$ is in $L^{1}[0, \infty)$.

We assume nothing about the norm of $a+a * \omega$ in $L^{1}[0, \infty)$. We now define $\eta(s)$ as

$$
\begin{aligned}
\eta(s) & =s-\alpha, & & s \geqq \frac{1}{2}(\alpha+\beta), \\
& =\beta-s, & & s \leqq \frac{1}{2}(\alpha+\beta) .
\end{aligned}
$$

$\eta(s)$ is a measure of the deviation from the "average" slope of $g$.

Equation (3.1) can be rewritten as

$$
x=f+\gamma a * x+a *(g(x)-\gamma x)
$$

where $\gamma$ is a constant to be specified later. Picking a function $\omega(t)$ which satisfies hypothesis (P8), we define

$$
w_{\gamma}(t)=\gamma(a(t)+\omega * a(t))-\omega(t) .
$$

Clearly $w_{y}(t) \in L^{1}[0, \infty)$ for all constants $\gamma$. Let $W_{\gamma}(s)$ be the Laplace transform of $w_{y}(t)$ and we assume

(P9) There is at least one $\gamma$ such that $W_{\gamma}(s) \neq 1$ for $\operatorname{Re} s \geqq 0$.

Let $\Gamma=\left\{\gamma \in R: W_{\gamma}(s) \neq 1\right.$ for $\left.\operatorname{Re} s \geqq 0\right\}$. By (P9), $\Gamma$ is nonvoid.

We now define, for $\gamma \in \Gamma$

$$
D(t) \equiv \gamma(a(t)+a * \omega(t))-\omega(t), \quad E(t) \equiv a(t)+a * \omega(t) .
$$


By the choice of $\omega(t)$, both functions are in $L^{1}[0, \infty)$. By the definition of $\Gamma$ and the Paley-Wiener theorem, the resolvent of $D(t)$ exists and is in $L^{1}[0, \infty)$. Let $F(t)=$ res $D(t)$. That is, there is a function $F$ in $L^{1}[0, \infty)$ such that $(I-D)^{-1}=I-F$. Finally, we pick $\gamma^{*}$ so as to minimize $\eta(\gamma)\|E-E * F\|_{B}$ over all $\gamma \in \Gamma$. While this minimum may not exist (since $\Gamma$ is not necessarily closed) we can approximate it as closely as desired.

TheOREM 3.1. Let $B$ be any of the spaces $B C[0, \infty), L^{p}[0, \infty), 1 \leqq p \leqq \infty$. Suppose $g$ maps $B \rightarrow B, f \in B$ and (P7)-(P9) are satisfied. Assume further that

$$
\eta\left(\gamma^{*}\right)\|E-E * F\|_{1}<1 .
$$

Then there exists a unique solution to (3.1) lying in $B$ for any $f$ in $B$.

Proof. Let $\gamma=\gamma^{*}$ in equation (3.2). As before, we define $v(t)$ by $v(t)=-\omega(t)$ $-\int_{0}^{t} \omega(t-s) v(s) d s$. Clearly, $(I+v)^{-1}=(I+w)$. Adding $v * x$ to both sides of equation (3.2), we obtain

$$
x+v * x=f+\left(\gamma^{*} a+v\right) * x+a *\left(g(x)-\gamma^{*}(x)\right)
$$

which implies that

$$
x=(I+v)^{-1} * f+(I+v)^{-1} *\left(\gamma^{*} a+v\right) * x+(I+v)^{-1} * a *\left(g(x)-\gamma^{*} x\right) .
$$

Now, if we let $h(t)=(I+v)^{-1} * f(t)$, it is apparent that $h(t) \in B$ since $f(t)+\omega * f(t)$ $\in B$. Also, $\quad(I+v)^{-1} *\left(\gamma^{*} a+v\right)=(I+\omega) *\left(\gamma^{*} a+v\right)=\gamma^{*} \omega * a+\gamma^{*} a+v * \omega+v=$ $\gamma^{*}(a+a * \omega)-\omega=D$. Similarly, $\left((I+v)^{-1} * a=(I+\omega) * a=a+a * \omega=E\right.$.

Thus equation (3.3) has become

$$
x=h+D * x+E *\left(g(x)-\gamma^{*} x\right)
$$

where $D$ and $E$ are in $L^{1}[0, \infty)$. Rewriting again, we have

$$
x-D * x=h+E *\left(g(x)-\gamma^{*} x\right)
$$

or

$$
x=(I-D)^{-1} * h+(I-D)^{-1} * E *\left(g(x)-\gamma^{*} x\right) .
$$

But $(I-D)^{-1}=I-F \in L^{1}[0, \infty)$. Let $m(t)=(I-F) * h(t) \in B$. Hence

$$
x=m+(E-E * F) *\left(g(x)-\gamma^{*} x\right) \equiv T x .
$$

We now show that $T$ is a contraction on $B$. Since $g$ maps $B \rightarrow B$, and $E-E * F$ $\in L^{1}[0, \infty$ ), the assumption that $m \in B$ (which is implied by having $f \in B$ ) implies that $T$ maps $B \rightarrow B$. Then

$$
\begin{aligned}
\left\|T x_{1}-T x_{2}\right\|_{B} & \leqq\|E-E * F\|_{1}\left\|g\left(x_{1}, s\right)-g\left(x_{2}, s\right)-\gamma^{*}\left(x_{1}-x_{2}\right)\right\|_{B} \\
& \leqq\|E-E * F\|_{1}\left\|\left(\frac{g\left(x_{1}, s\right)-g\left(x_{2}, s\right)}{x_{1}-x_{2}}-\gamma^{*}\right)\left(x_{1}-x_{2}\right)\right\|_{B} \\
& \leqq\|E-E * F\|_{1} \eta\left(\gamma^{*}\right)\left\|x_{1}-x_{2}\right\|_{B}<\left\|x_{1}-x_{2}\right\|_{B} .
\end{aligned}
$$

Q.E.D. 
Finding examples of the implementation of Theorem 3.1 is fairly easy. We have already encountered examples of finding an $\omega$ which satisfies $a+a * \omega \in L^{1}[0, \infty)$ when $a(t)$ only lies in $L L^{1}[0, \infty)$.

We are thus left with the problem of determining whether the nonlinear term has the right slope restrictions and if $\eta\left(\gamma^{*}\right)$ is small enough. One would suppose that in many practical examples, $\eta\left(\gamma^{*}\right)\|E-E * F\|_{1}$ will not necessarily be $<1$. It is still possible, however, to derive an existence-uniqueness theorem when the forcing function $f$ lies in a suitably small ball about the origin. We need the following assumption:

(P10) For all $\delta>0$, there exists an $\varepsilon>0$ such that if $\|x\|_{B},\|y\|_{B}<\varepsilon, \alpha(x-y)$ $\leqq g(x, t)-g(y, t) \leqq \beta(x-y)$ where $\beta-\alpha<\delta$.

Under this assumption, $\eta\left(\gamma^{*}\right)$ can be made as small as desired by restriction to a suitably small ball in $B$.

Corollary 3.2. Suppose $g$ maps $B \rightarrow B, g(0, s) \equiv 0, f \in B$, and (P7), (P8), (P9) and (P10) are satisfied. Then there exist $\varepsilon_{1}, \varepsilon_{2}>0$ such that if $\|f\|_{B}<\varepsilon_{1}$, there exists a unique solution $x(t)$ to equation (3.1) lying in $B$ and $\|x\|_{B}<\varepsilon_{2}$.

Proof. As before, we write (3.1) as

$$
x=m+(E-E * F) *\left(g(x)-\gamma^{*} x\right) \equiv T x .
$$

$T$ will be a contraction if $\|E-E * F\|_{1} \eta\left(\gamma^{*}\right)<1$. Pick $\varepsilon_{2}$ so that $\eta\left(\gamma^{*}\right)\|E-E * F\|_{1}$ $<\frac{1}{2}$, which is possible by (P10). Let $S\left(0, \varepsilon_{2}\right)=\left\{x \in B:\|x\|_{B} \leqq \varepsilon_{2}\right\}$. We must show that $T$ maps $S\left(0, \varepsilon_{2}\right) \rightarrow S\left(0, \varepsilon_{2}\right)$. Pick $\varepsilon_{1}$ so that $\|f\|_{B} \leqq \varepsilon_{1}$ implies that $\|m\|_{B} \leqq \varepsilon_{2} / 2$. Then for $x \in S\left(0, \varepsilon_{2}\right)$,

$$
\begin{aligned}
\|T x\| & \leqq\|m\|+\|E-E * F\|\left\|g(x)-\gamma^{*} x\right\| \\
& \leqq \frac{\varepsilon_{2}}{2}+\|E-E * F\|\left\|\frac{g(x, s)-g(0, s)}{x-0}-\gamma^{*}\right\|\|x\| \\
& \leqq \frac{\varepsilon_{2}}{2}+\|E-E * F\| \eta\left(\gamma^{*}\right) \varepsilon_{2} \leqq \frac{\varepsilon_{2}}{2}+\frac{\varepsilon_{2}}{2}=\varepsilon_{2} .
\end{aligned}
$$

Examples of the application of this corollary are easily found. A general class of such examples are generated by the next result.

Corollary 3.3. Let $B=B C[0, \infty)$ and assume that $g(x, s)$ is differentiable in $x$ and satisfies $\partial g(x, s) / \partial x=O(\|x\|)$ as $\|x\| \rightarrow 0$. Then $(\mathrm{P} 10)$ is satisfied.

Proof. Obvious by the mean value theorem.

The preceding analysis is now extended to equations with several kernels. For example, consider the following nonlinear network: 


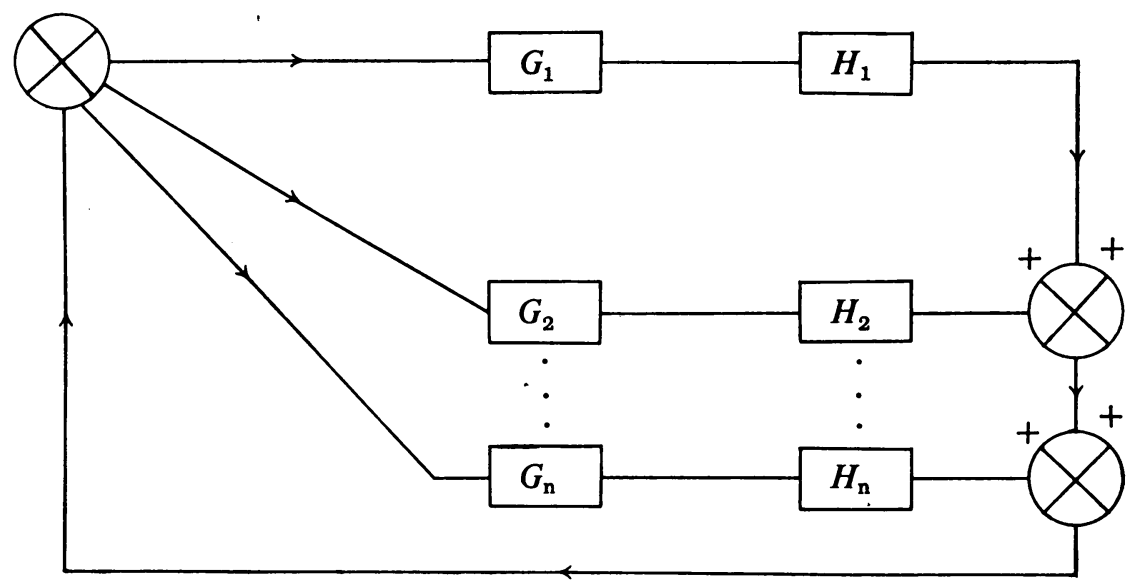

We can write the equations governing this system as

$$
x=f+H_{1} * G_{1}(x)+H_{2} * G_{2}(x)+\cdots+H_{n} * G_{n}(x) .
$$

We assume that $H_{i} \in L^{1}[0, \infty)$ for each $i$. If this were not the case, we could do an analysis similar to that done above for the one kernel case. We again stipulate that $G_{i}$ maps $B \rightarrow B$ (where $B$ is $B C[0, \infty)$ ) and

(P11) There exists $\alpha_{i}, \beta_{i}$ such that $\alpha_{i} \leqq\left(G_{i}(x, s)-G_{i}(y, s)\right) /(x-y) \leqq \beta_{i}$ and $\alpha_{i}<\beta_{i}$. As before, we define the functions $\eta_{i}(s)$ by

$$
\begin{aligned}
\eta_{i}(s) & =s-\alpha_{i}, & & s \geqq \frac{1}{2}\left(\alpha_{i}+\beta_{i}\right), \\
& =\beta_{i}-s, & & s \leqq \frac{1}{2}\left(\alpha_{i}+\beta_{i}\right) .
\end{aligned}
$$

To simplify matters we do our analysis for $n=2$. For $n>2$ the procedure will be the same. Thus our equation is

$$
x=f+H_{1} * G_{1}(x)+H_{2} * G_{2}(x) .
$$

We define the set $A$ by $A=\left\{a \in R: a H_{1}(s) \neq 1\right.$ for $\left.\operatorname{Re} s \geqq 0\right\}$ and assume that $A$ is nonvoid. We similarly define, for $a$ in $A, r_{a}=$ res $a H_{1}$ which exists and is $L^{1}[0, \infty)$ by the Paley-Wiener theorem. Since $f \in B$, we define $f_{1}=\left(I-r_{a}\right) * f \in B$. We similarly define, for $a$ in $A$,

$$
H_{10}=\left(I-r_{a}\right) * H_{1}, \quad H_{20}=\left(I-r_{a}\right) * H_{2} .
$$

Clearly $H_{10}$ and $H_{20}$ are in $L^{1}[0, \infty)$. We now define the set $C=\left\{c \in R: c H_{20}(s) \neq 1\right.$ for $\operatorname{Re} s \geqq 0\}$ and assume that $C$ is nonvoid. Finally, for $c \in C$ and $r_{c}=\operatorname{res} c H_{20}$ $\in L^{1}[0, \infty)$ we define

$$
f_{2}=\left(I-r_{c}\right) * f_{1}, \quad H_{11}=\left(I-r_{c}\right) * H_{10}, \quad H_{21}=\left(I-r_{c}\right) * H_{20} .
$$

Obviously $f_{2} \in B$ and $H_{11}, H_{21} \in L^{1}[0, \infty)$.

We are now ready to state the companion result to Theorem 3.1 for systems. 
THEOREM 3.4. Let $H_{i}(t) \in L^{1}[0, \infty)$ for $i=1,2$ and suppose $G_{i}$ maps $B \rightarrow B$ and satisfies (P11). Assume further that there exist $a^{*} \in A$ and $c^{*} \in C$ such that $\left[\left\|H_{11}\right\| \eta_{1}\left(a^{*}\right)+\left\|H_{21}\right\| \eta_{2}\left(c^{*}\right)\right]<1$. Then for any $f \in B$, there exists $a$ unique solution to equation (3.6) in $B$.

Proof. Proceeding as before, we write (3.6) as

$$
x=f+a^{*} H_{1} * x+H_{1} *\left[G_{1}(x)-a^{*} x\right]+H_{2} *\left[G_{2}(x)\right]
$$

or

$$
x-a^{*} H_{1} * x=f+H_{1} *\left[G_{1}(x)-a^{*} x\right]+H_{2} * G_{2}(x)
$$

or

$$
\begin{aligned}
x & =f_{1}+H_{10} *\left[G_{1}(x)-\alpha^{*} x\right]+H_{20} * G_{2}(x) \\
& =f_{1}+H_{10} *\left[G_{1}(x)-a^{*} x\right]+c^{*} H_{20} * x+H_{20} *\left[G_{2}(x)-c^{*} x\right]
\end{aligned}
$$

or

$$
x-c^{*} H_{20} * x=f_{1}+H_{10} *\left[G_{1}(x)-a^{*} x\right]+H_{20} *\left[G_{2}(x)-c^{*} x\right]
$$

or

$$
x=f_{2}+H_{11} *\left[G_{1}(x)-a^{*} x\right]+H_{21} *\left[G_{2}(x)-c^{*} x\right] \equiv T x .
$$

Clearly $T$ maps $B \rightarrow B$. To show that $T$ is a contraction, note that

But

$$
\begin{aligned}
\left\|T x_{1}-T x_{2}\right\| \leqq & \left\|H_{11}\right\|\left\|G_{1}\left(x_{1}\right)-G_{1}\left(x_{2}\right)-a^{*}\left(x_{1}-x_{2}\right)\right\| \\
& +\left\|H_{21}\right\|\left\|G_{2}\left(x_{1}\right)-G_{2}\left(x_{2}\right)-c^{*}\left(x_{1}-x_{2}\right)\right\| .
\end{aligned}
$$

$$
\begin{aligned}
\left\|G_{1}(x)-G_{1}\left(x_{2}\right)-a^{*}\left(x_{1}-x_{2}\right)\right\| & =\left\|\left(\frac{G_{1}\left(x_{1}\right)-G_{1}\left(x_{2}\right)}{x_{1}-x_{2}}-a^{*}\right)\left(x_{1}-x_{2}\right)\right\| \\
& \leqq \eta_{1}\left(a^{*}\right)\left\|x_{1}-x_{2}\right\|
\end{aligned}
$$

and similarly for $G_{2}$. Thus,

$$
\left\|T x_{1}-T x_{2}\right\| \leqq\left[\left\|H_{11}\right\| \eta_{1}\left(a^{*}\right)+\left\|H_{21}\right\| \eta_{2}\left(c^{*}\right)\right]\left\|x_{1}-x_{2}\right\|<\left\|x_{1}-x_{2}\right\| \text {. Q.E.D. }
$$

NoTe. If $\left\|H_{11}\right\| \eta_{1}\left(a^{*}\right)+\left\|H_{21}\right\| \eta_{2}\left(c^{*}\right)>1$, we can still find a solution to (3.6) if we restrict $f$ as in Corollary 3.2.

\section{BIBLIOGRAPHY}

1. V. E. Beneš and I. W. Sandberg, On the response of time-variable nonlinear systems to almost periodic signals, J. Math. Anal. Appl. 10 (1965), 245-268. MR 30 \#5856.

2. C. Corduneanu, Some perturbation problems in the theory of integral equations, Math. Systems Theory 1 (1967), 143-155. MR 35 \#4773.

3. R. K. Miller, On Volterra integral equations with nonnegative integrable resolvents, J. Math. Anal. Appl. 22 (1968), 319-340. MR 37 \#3291.

4. - On the linearization of Volterra integral equations, J. Math. Anal. Appl. 23 (1968), 198-208. MR 37 \#5635.

5. R. K. Miller, J. A. Nohel and J. S. W. Wong, Perturbations of Volterra integral equations, J. Math. Anal. Appl. 25 (1969), 676-691. 
6. John A. Nohel, Remarks on nonlinear Volterra equations, Proc. United States-Japan Seminar Differential and Functional Equations (Minneapolis, Minn., 1967), Benjamin, New York, 1967, pp. 249-266. MR 36 \#5636.

7. R. E. A. C. Paley and N. Wiener, Fourier transforms in the complex domain, Amer. Math. Soc. Colloq. Publ., vol. 19, Amer. Math. Soc., Providence, R. I., 1934; reprint, 1967.

8. I. W. Sandberg, On the boundedness of solutions of nonlinear integral equations, Bell System Tech. J. 44 (1965), 439-453. MR 33 \#7806.

9. D. V. Widder, The Laplace transform, Princeton Math. Series, vol. 6, Princeton Univ. Press, Princeton, N. J., 1941. MR 3, 232.

10. G. Zames, On the input-output stability of time-varying nonlinear feedback systems, IEEE Trans. Automatic Control AC-11 (1966), 228-238, 465-476.

MCGILl UNIVERSITY, Montreal, Canada 